\title{
Another Type of Cauchy's Integral Formula in Complex Clifford Analysis
}

\author{
Kimirô SANO \\ Hachinohe Institute of Technology \\ (Communicated by T. Suzuki)
}

\begin{abstract}
We prove Cauchy's integral formula for complex regular functions in complex Clifford analysis. We claim that our formula is valid in any dimension. Furthermore, we study some properties of manifolds where the integration in the formula is defined and relax the conditions imposed on the manifolds.
\end{abstract}

\section{Introduction.}

The aim of this paper is to present Cauchy's integral formula in complex Clifford analysis, which is different from that in [7].

In [2] Delanghe et al. gave Cauchy's integral formula for regular (monogenic in their terminology) functions in real Clifford analysis. Ryan $[4,5,6]$, Bureš and Souček $[3,8]$ complexified this result and gave Cauchy's integral formula for complex regular functions in complex Clifford analysis. However, they restricted the arguments only to even dimension. More recently the author [7] gave a homological version of Cauchy's integral formula for complex regular functions, which is valid in any dimension; this generalizes Souček and Bureš' results.

On the other hand, there has been no generalization of Ryan's results. In this paper we prove the following Cauchy integral formula; this generalizes Ryan's results.

THEOREM. Let $p, q \in \mathbf{N}$ with $p \leqq q, \mathbf{C}_{p}$ be the universal Clifford algebra for $\mathbf{C}^{p}$; then $\mathbf{C}^{p+1} \subset \mathbf{C}_{p}$ (see $\left.[2, \S 1]\right)$. Let $f$ be a complex right regular function in a domain $\Omega$ of $\mathbf{C}^{p+1}$ with values in $\mathbf{C}_{q}$. Let $a \in \Omega$ and $M$ be a real $(p+1)$-dimensional piecewise smooth compact connected oriented submanifold of $\Omega$ with boundary such that $a \in M^{\circ}, M$ is smooth at a, $M \cap \Lambda_{a}=\{a\}$ and $T_{a} M \cap \Lambda_{0}=\{0\}$. Further, suppose that $\langle w-a\rangle^{p-1}$ is single-valued with respect to $w$ in $M$. Then

$$
f(z)=\frac{1}{\left|S^{p}\right|} \int_{\partial M} f(w) d \sigma_{w} \frac{1}{(w-z)\langle w-z\rangle^{p-1}} \quad \text { for any } z \in D_{\Omega}(\partial M, a),
$$


where $\left|S^{p}\right|$ is the area of the $p$-dimensional unit sphere, $\langle w-z\rangle=\left(\sum_{j=0}^{p}\left(w_{j}-z_{j}\right)^{2}\right)^{1 / 2}$, $1 /(w-z)$ is the inverse element of a Clifford number $w-z \in \mathbf{C}^{p+1} \subset \mathbf{C}_{p}, d \sigma_{w}$ is the $\mathbf{C}_{p}$-valued surface-element (see $\left[4\right.$, p. 137]), $\Lambda_{z}=\left\{w \in C^{p+1} \mid\langle w-z\rangle=0\right\}$ and $D_{\Omega}(\partial M, a)$ is the component of $\Omega \backslash \bigcup_{z \in \partial M} \Lambda_{z}$ which contains $a$.

According to this result we no longer need Ryan's concept of manifolds of type two and others (see [6, pp. 38-39]).

To obtain these results we study $R$-linear subspaces of $\mathbf{C}^{p+1}$. In $\S 2$ we fix the notation and define complex regular functions. In $\$ 3$ we study the complex vector space $\mathrm{C}^{p+1}$ with the complex inner product $z \cdot w=\sum_{j=0}^{p} z_{j} w_{j}$. In $\S 4$ we investigate oriented $\mathbf{R}$-linear subspaces of $\mathbf{C}^{p+1} \cong \mathbf{R}^{2 p+2}$ in the real Grassmannians. Finally, in $\S 5$ we prove Cauchy's integral formula for complex regular functions in every dimension (Theorems $4,5)$.

\section{Preliminaries.}

Let $p \in \mathbf{N}$ and $\mathbf{R}_{p}$ be the universal Clifford algebra constructed from the space $\mathbf{R}^{p}$ with orthonormal basis $\left\{\mathbf{e}_{1}, \cdots, \mathbf{e}_{p}\right\}$. Furthermore, let $\mathbf{e}_{0}=1$ be the unity of $\mathbf{R}_{p}$; then $\mathbf{e}_{0}, \mathbf{e}_{1}, \cdots, \mathbf{e}_{p}, \mathbf{e}_{1} \mathbf{e}_{2}, \cdots, \mathbf{e}_{1} \cdots \mathbf{e}_{p}$ are basis elements of $\mathbf{R}_{p}$ such that

$$
\mathbf{e}_{j} \mathbf{e}_{k}+\mathbf{e}_{k} \mathbf{e}_{j}=-2 \delta_{j, k} \quad \text { for } 1 \leqq j, k \leqq p .
$$

By $\mathbf{C}_{p}$ we denote the universal complex Clifford algebra $\mathbf{R}_{p} \otimes_{\mathbf{R}} \mathbf{C}$. Furthermore, we set $\mathbf{R}_{0}=\mathbf{R}$ and $\mathbf{C}_{0}=\mathbf{C}$. For any $a \in \mathbf{C}_{p}$ we write

$$
a=\sum_{k=0}^{p} \sum_{j_{1}<\cdots<j_{k}} a_{j_{1}, \cdots, j_{k}} \mathbf{e}_{j_{1}} \cdots \mathbf{e}_{j_{k}},
$$

where $j_{1}, \cdots, j_{k} \in\{1, \cdots, p\}, a_{j_{1}}, \cdots, j_{k} \in \mathbf{C}$ and $a_{\varnothing}=a_{0}$. We define a norm on $\mathbf{C}_{p}$ as $|a|^{2}=\sum_{k=0}^{p} \sum_{j_{1}<\cdots<j_{k}}\left|a_{j_{1}}, \cdots, j_{k}\right|^{2} ;$ then we have the topological isomorphisms $\mathbf{R}_{p} \cong \mathbf{R}^{2^{p}}$ and $\mathbf{C}_{p} \cong \mathbf{C}^{2^{p}}$. The elements $x=\left(x_{0}, \cdots, x_{p}\right) \in \mathbf{R}^{p+1}$ and $z=\left(z_{0}, \cdots, z_{p}\right) \in \mathbf{C}^{p+1}$ will be identified respectively with the Clifford numbers $x=\sum_{j=0}^{p} x_{j} \mathbf{e}_{j}$ and $z=\sum_{j=0}^{p} z_{j} \mathbf{e}_{j}$. Moreover, we set $\tilde{z}=z_{0}-\sum_{j=1}^{p} z_{j} \mathbf{e}_{j}$, then we have $1 / z=\tilde{z} /\left(\sum_{j=0}^{p} z_{j}^{2}\right)$.

Let $\Omega$ be a domain of $\mathbf{C}^{p+1}, f: \Omega \rightarrow \mathbf{C}_{q}(p \leqq q)$ be holomorphic and $D_{z}=\sum_{j=0}^{p} \mathbf{e}_{j}\left(\partial / \partial z_{j}\right)$ stand for the complex Cauchy-Riemann operator. Then $f$ is said to be complex left (resp. right) regular in $\Omega$ if $D_{z} f=0$ (resp. $f D_{z}=0$ ) in $\Omega$ (cf. $[2, \S 8]$ ).

We define the $\mathbf{C}_{\boldsymbol{p}}$-valued surface-element as

$$
d \sigma_{z}=\sum_{j=0}^{p}(-1)^{j} \mathbf{e}_{j} d z_{0} \wedge \cdots \wedge d \hat{z}_{j} \wedge \cdots \wedge d z_{p},
$$

where $d \hat{z}_{j}$ means that $d z_{j}$ has to be omitted. For $z, w \in \mathbf{C}^{p+1}$ we set $z \cdot w=[z \tilde{w}]_{0}=$ $\sum_{j=0}^{p} z_{j} w_{j}$ and write $Q(z)=z \tilde{z}=z \cdot z$ and $\langle z\rangle=\sqrt{Q(z)}$, where $[z \tilde{w}]_{0}$ is the coefficient of $\mathbf{e}_{0}$ in $z \tilde{w}$. Furthermore, we write $\Lambda_{z}=\left\{w \in \mathbf{C}^{p+1} \mid Q(w-z)=0\right\}$ and $\Lambda(K)=\bigcup_{z \in K} \Lambda_{z}$ for 
a set $K \subset \mathbf{C}^{p+1}$. By $[z, w]$ we denote the line-segment joining $z$ to $w$.

By $\mathscr{G}_{k}\left(\mathbf{C}^{n}, \mathbf{R}\right)$, or simply by $\mathscr{G}_{k}\left(\mathbf{C}^{n}\right)$ we denote the real Grassmannian consisting of all oriented real $k$-dimensional linear subspaces of $\mathbf{C}^{n}$. For $U \in \mathscr{G}_{k}\left(\mathbf{C}^{n}\right),-U$ stands for the oppositely oriented linear subspace. For a smooth manifold $M$ we denote by $T M$ the tangent bundle of $M$.

Let $\operatorname{Mat}(m, n, \mathbf{C})$ be the set of all $(m, n)$-matrices over $\mathbf{C}, I$ be the unit matrix and $\left|S^{p}\right|$ be the area of the $p$-dimensional unit sphere $S^{p}$. We set $\mathbf{N}_{0}=\mathbf{N} \cup\{0\}$ and $\mathbf{R}_{+}=\{x \in \mathbf{R} \mid x \geqq 0\}$. In the sequel, we assume that the vertex of a cone $L$ in $\mathbf{C}$ is the origin 0 .

Set $G=\left\{w \in \mathbf{C}^{p+1} \mid Q(w)>0\right\}$ and consider the disjoint union $G C^{1}=\bigcup_{0 \leqq \theta<2 \pi} G e^{i \theta}$, where $C^{1}$ is the unit circle in $\mathbf{C e}_{0}(=\mathbf{C})$; put $V_{0}^{p+1}=G C^{1} \cup \Lambda_{0}$ and $V_{z}^{p+1}=z+V_{0}^{p+1}$. Then $V_{z}^{p+1}$ is a $(p+1)$-dimensional complex manifold on which $\langle w-z\rangle$ is single-valued with respect to $w$ (cf. [7]); note that $\mathbf{R}^{p+1} \subset V_{0}^{p+1}$. By $S^{p}$ we denote the unit $p$-sphere in $\mathbf{R}^{p+1}$; note that $\left.\langle w\rangle\right|_{S^{p}}=|w|$. Furthermore, by $\pi_{z}$ we denote the map which associates to each point $w \in V_{z}^{p+1}$ the point $w \in \mathbf{C}^{p+1}$. Since $\langle w-z\rangle$ is double-valued in $\mathbf{C}^{p+1} \backslash \Lambda_{z}$, each point $w \in \mathbf{C}^{p+1} \backslash \Lambda_{z}$ is associated to two points $w \in V_{z}^{p+1} \backslash \Lambda_{z}$. For a domain $\Omega$ of $\mathrm{C}^{p+1}$ we write $\Omega_{z}=\pi_{z}^{-1}(\Omega)\left(\subset V_{z}^{p+1}\right)$.

\section{The complex quadratic form $Q(z)$.}

In this section we study some properties of the complex quadratic form $Q(z)$. Moreover, we give Schmidt's orthonormalization of a finite set of complex vectors with respect to the complex inner product $z \cdot w$.

Definition 1. Let $p \in \mathbf{N}_{0}$ and $u \in \mathbf{C}^{p+1}$. If $Q(u) \neq 0$ or $u=0$, then $u$ is said to be non-degenerate with respect to $Q$.

REMARK. If $p=0$, then all $u \in \mathbf{C}$ are non-degenerate with respect to $Q$.

Definition 2. Let $p \in \mathbf{N}_{0}$ and $u_{1}, \cdots, u_{n} \in \mathbf{C}^{p+1}$. If $Q\left(x_{1} u_{1}+\cdots+x_{n} u_{n}\right)=0$ for $x_{1}, \cdots, x_{n} \in \mathbf{R}$ implies $x_{1}=\cdots=x_{n}=0$, then $\left\{u_{1}, \cdots, u_{n}\right\}$ is said to be linearly independent over $\mathbf{R}$ with respect to $Q$.

REMARK. If we replace $Q(z)$ by $|z|^{2}$ in Definition 2 , then $\left\{u_{1}, \cdots, u_{n}\right\}$ is linearly independent over $\mathbf{R}$ in ordinary sense.

By Definitions 1 and 2 we immediately obtain the following.

Proposition 1. Let $p \in \mathbf{N}_{0}$ and $\left\{u_{1}, \cdots, u_{n}\right\} \subset \mathbf{C}^{p+1}$ be linearly independent over $\mathbf{R}$ with respect to $Q$; set $U=\mathbf{R} u_{1}+\cdots+\mathbf{R} u_{n}$. If $\left\{v_{1}, \cdots, v_{m}\right\} \subset U(m \leqq n)$ is linearly independent over $\mathbf{R}$, then it is linearly independent over $\mathbf{R}$ with respect to $Q$.

Proposition 2. Let $p \in \mathbf{N}_{0}$; then $\left\{u_{1}, \cdots, u_{n}\right\} \subset \mathbf{C}^{p+1}$ is linearly independent over $\mathbf{R}$ with respect to $Q$ if and only if it is linearly independent over $\mathbf{R}$ and $x_{1} u_{1}+\cdots+x_{n} u_{n}$ 
is non-degenerate with respect to $Q$ for any $x_{1}, \cdots, x_{n} \in \mathbf{R}$.

REMARK. Let $p=0$; then $\left\{u_{1}, \cdots, u_{n}\right\} \subset \mathbf{C}$ is linearly independent over $\mathbf{R}$ with respect to $Q$ if and only if it is linearly independent over $\mathbf{R}$.

Definition 3. Let $p \in \mathbf{N}_{0}$ and $\left\{u_{1}, u_{2}\right\} \subset \mathbf{C}^{p+1}$ be linearly independent over $\mathbf{R}$ with respect to $Q$; set $F:[0,2 \pi] \ni \theta \mapsto Q\left(u_{1} \cos \theta+u_{2} \sin \theta\right) \in \mathbf{C} \backslash\{0\}$. Then we define the sign of the vectors $\left\{u_{1}, u_{2}\right\}$ with respect to $Q$ as $\operatorname{sgn}\left(u_{1}, u_{2}\right)=\frac{1}{2} \lambda$, where $\lambda$ is the winding number of $F$ with respect to 0 , that is, $\lambda=0, \pm 2$.

Proposition 3. Let $p \in \mathbf{N}, 3 \leqq n$ and $\left\{u_{1}, \cdots, u_{n}\right\} \subset \mathbf{C}^{p+1}$ be linearly independent over $\mathbf{R}$ with respect to $Q$; set $U=\mathbf{R} u_{1}+\cdots+\mathbf{R} u_{n}$. If $\left\{v_{1}, v_{2}\right\} \subset U$ is linearly independent over $\mathbf{R}$, then $\operatorname{sgn}\left(v_{1}, v_{2}\right)=0$.

ProOF. There are vectors $v_{3}, \cdots, v_{n} \in U$ such that $\left\{v_{1}, \cdots, v_{n}\right\}$ is linearly independent over $\mathbf{R}$. Since $\left\{u_{1}, \cdots, u_{n}\right\}$ is linearly independent over $\mathbf{R}$ with respect to $Q$, from Proposition 1 it follows that $\left\{v_{1}, \cdots, v_{n}\right\}$ is linearly independent over $\mathbf{R}$ with respect to $Q$. Set

$$
S=\left\{x_{1} v_{1}+\cdots+x_{n} v_{n}|| x \mid=1, x_{1}, \cdots, x_{n} \in \mathbf{R}\right\}
$$

and $C:[0,2 \pi] \ni \theta \mapsto v_{1} \cos \theta+v_{2} \sin \theta \in S$; then we obtain $S \cap \Lambda_{0}=\varnothing$. Since $n \geqq 3, C$ is homotopic to zero in $S$; therefore, $Q(C)$ is also homotopic to zero in $\mathbf{C} \backslash\{0\}$, that is, $\operatorname{sgn}\left(v_{1}, v_{2}\right)=0$.

PROPOSITION 4. Let $p \in \mathbf{N}_{0}$ and $\left\{u_{1}, \cdots, u_{n}\right\} \subset \mathbf{C}^{p+1}$ be linearly independent over $\mathbf{R}$ with respect to $Q$; set $U=\mathbf{R} u_{1}+\cdots+\mathbf{R} u_{n}$.

(i) If $n \neq 2$, then $L=Q(U)$ is a closed convex cone in C such that $L \cap(-L)=\{0\}$.

(ii) If $n=2$ and if $\operatorname{sgn}\left(u_{1}, u_{2}\right)=0$, then $L=Q(U)$ is a closed convex cone in $\mathbf{C}$ such that $L \cap(-L)=\{0\}$.

(iii) If $n=2$ and if $\operatorname{sgn}\left(u_{1}, u_{2}\right) \neq 0$, then $Q(U)=\mathbf{C}$.

ProOF. (i): We have only to show that the assertion is true for $n \geqq 3$. It is clear that $L$ is a closed cone in $C$; therefore, we show that $L$ is convex and that $L \cap(-L)=\{0\}$. Let $w_{1}, w_{2} \in L \backslash\{0\}$; then if $L=\mathbf{R}_{+} w_{1}$, the assertion is clearly true. Suppose that $w_{2} \notin \mathbf{R}_{+} w_{1}$ and let $v_{1}, v_{2} \in U$ satisfy $Q\left(v_{j}\right)=w_{j}$ for $j=1,2$; then $\left\{v_{1}, v_{2}\right\}$ is linearly independent over R. Therefore, by Proposition 3, we obtain $\operatorname{sgn}\left(v_{1}, v_{2}\right)=0$. Set

$$
C:[0,2 \pi] \ni \theta \mapsto v_{1} \cos \theta+v_{2} \sin \theta \in U \backslash\{0\} ;
$$

then $w_{1}, w_{2} \in Q(C) \subset L$ and the winding number of the ellipse $Q(C)$ with respect to 0 is 0 . Thus, we conclude that $w_{2} \neq-w_{1}$, that is, $L \cap(-L)=\{0\}$. Furthermore, since $L$ is a cone with $Q(C) \subset L$, we find that $\left[w_{1}, w_{2}\right] \subset L$, that is, $L$ is convex.

(ii): We use the same notation as above. We have only to show that $\operatorname{sgn}\left(v_{1}, v_{2}\right)=0$ without using Proposition 3. Set 


$$
C_{1}:[0,2 \pi] \ni \theta \mapsto u_{1} \cos \theta+u_{2} \sin \theta \in U \backslash\{0\} ;
$$

then since $U \cap \Lambda_{0}=\{0\}$ and since $C$ is homotopic to $C_{1}$ or to the oppositely oriented loop $-C_{1}$ in $U \backslash\{0\}, Q(C)$ is also homotopic to $\pm Q\left(C_{1}\right)$ in $\mathbf{C} \backslash\{0\}$. Thus, the assumption implies $\operatorname{sgn}\left(v_{1}, v_{2}\right)= \pm \operatorname{sgn}\left(u_{1}, u_{2}\right)=0$.

(iii): From Definition 3 the assertion immediately follows.

Q.E.D.

PROPOSITION 5. Let $p \in \mathbf{N}_{0}$ and $\left\{u_{1}, \cdots, u_{n}\right\} \subset \mathbf{C}^{p+1}$ be linearly independent over $\mathbf{R}$ with respect to $Q$.

(i) If $n \neq 2$, then $\left\{u_{1}, \cdots, u_{n}\right\}$ is linearly independent over $\mathbf{C}$.

(ii) If $n=2$ and if $\operatorname{sgn}\left(u_{1}, u_{2}\right)=0$, then $\left\{u_{1}, u_{2}\right\}$ is linearly independent over $\mathbf{C}$.

Proof. Set $U=\mathbf{R} u_{1}+\cdots+\mathbf{R} u_{n}$ and $z_{j}=x_{j}+i y_{j}$, where $x_{j}, y_{j} \in \mathbf{R}$ for $j=1, \cdots, n$. Suppose $z_{1} u_{1}+\cdots+z_{n} u_{n}=0$; then we have

$$
x_{1} u_{1}+\cdots+x_{n} u_{n}+i\left(y_{1} u_{1}+\cdots+y_{n} u_{n}\right)=0 .
$$

Set $v=x_{1} u_{1}+\cdots+x_{n} u_{n}$; then by (1) we obtain $i v \in U$ and therefore, $-Q(v)=Q(i v) \in Q(U)$. Moreover, according to the assumption and Proposition 4 (i) or (ii), we obtain

$$
Q(v)=Q\left(x_{1} u_{1}+\cdots+x_{n} u_{n}\right)=0 .
$$

Hence, from the assumption and (1) we conclude that $x_{1}=\cdots=x_{n}=y_{1}=\cdots=y_{n}=0$, that is, $z_{1}=\cdots=z_{n}=0$; this indicates that $\left\{u_{1}, \cdots, u_{n}\right\}$ is linearly independent over C.

Q.E.D.

CoRollaRy 1. Let $p \in \mathbf{N}_{0},\left\{u_{1}, \cdots, u_{n}\right\} \subset \mathbf{C}^{p+1}$ be linearly independent over $\mathbf{R}$ with respect to $Q$. Then

(i) $p=0 \Rightarrow n=1,2$.

(ii) $p \geqq 1 \Rightarrow 1 \leqq n \leqq p+1$.

ProOF. (i): From the remark after Proposition 2 the assertion follows.

(ii): By Proposition 2 we have $n \leqq 2 p+2$; if $p+2 \leqq n$, then Proposition 5 (i) implies that $\left\{u_{1}, \cdots, u_{n}\right\}$ is linearly independent over $\mathbf{C}$. However, this is impossible because $\left\{u_{1}, \cdots, u_{n}\right\} \subset \mathbf{C}^{p+1}$; thus, we conclude that $n \leqq p+1$.

Q.E.D.

Proposition 6 (Schmidt's orthonormalization). Let $p \in \mathbf{N}_{0}$ and $\left\{u_{1}, \cdots, u_{n}\right\} \subset$ $\mathbf{C}^{p+1}$ be linearly independent over $\mathbf{R}$ with respect to $Q$. When $n=2$, suppose further that $\operatorname{sgn}\left(u_{1}, u_{2}\right)=0$. Then an orthonormal system $\left\{v_{1}, \cdots, v_{n}\right\} \subset \mathbf{C} u_{1}+\cdots+\mathbf{C} u_{n}$ with respect to the complex inner product $z \cdot w$ is constructed in the following way.

$$
\begin{array}{cc}
v_{1}=u_{1} /\left\langle u_{1}\right\rangle . & \\
v_{2}=v_{2}^{\prime} /\left\langle v_{2}^{\prime}\right\rangle, & v_{2}^{\prime}=u_{2}-\left(u_{2} \cdot v_{1}\right) v_{1} . \\
\vdots & \vdots \\
v_{n}=v_{n}^{\prime} /\left\langle v_{n}^{\prime}\right\rangle, & v_{n}^{\prime}=u_{n}-\left(u_{n} \cdot v_{1}\right) v_{1}-\cdots-\left(u_{n} \cdot v_{n-1}\right) v_{n-1} .
\end{array}
$$


Moreover, $\left\{v_{1}, \cdots, v_{n}\right\}$ is linearly independent over $\mathbf{C}$.

REMARK. Each $v_{j}^{\prime}$ is determined independent of the choice of the value of $\left\langle v_{j}^{\prime}\right\rangle$.

Proof. If $\left\{v_{1}, \cdots, v_{n}\right\}$ is orthonormal with respect to $z \cdot w$, then it is linearly independent over $\mathrm{C}$. Thus, it is sufficient to show that $Q\left(u_{1}\right) \neq 0$ and $Q\left(v_{j}^{\prime}\right) \neq 0$ for any $j=2, \cdots, n$; by the assumption we have $Q\left(u_{1}\right) \neq 0$.

When $j=2$, suppose that $Q\left(v_{2}^{\prime}\right)=0$; then since $v_{2}^{\prime} \cdot u_{1}=v_{2}^{\prime} \cdot v_{1}=0$ and $u_{2}=$ $\left(u_{2} \cdot v_{1}\right) v_{1}+v_{2}^{\prime}$, we obtain

$$
Q\left(x_{1} u_{1}+x_{2} u_{2}\right)=Q\left(x_{1} u_{1}+x_{2}\left(u_{2} \cdot v_{1}\right) v_{1}\right) \quad \text { for any } x_{1}, x_{2} \in \mathbf{R} .
$$

Therefore, according to the assumption, $\left\{u_{1},\left(u_{2} \cdot v_{1}\right) v_{1}\right\}$ is linearly independent over $\mathbf{R}$ with respect to $Q$ with $\operatorname{sgn}\left(u_{1}, u_{2}\right)=\operatorname{sgn}\left(u_{1},\left(u_{2} \cdot v_{1}\right) v_{1}\right)$. If $n=2$, then by the assumption we have $\operatorname{sgn}\left(u_{1}, u_{2}\right)=0$; if $n \geqq 3$, then by the assumption and Proposition 3 we obtain $\operatorname{sgn}\left(u_{1}, u_{2}\right)=0$. Hence, Proposition 5 (ii) implies that $\left\{u_{1},\left(u_{2} \cdot v_{1}\right) v_{1}\right\}$ is linearly independent over $\mathrm{C}$; however, this is impossible. Thus, we infer that $Q\left(v_{2}^{\prime}\right) \neq 0$.

When $j=3, \cdots, n$, suppose that $Q\left(v_{j}^{\prime}\right)=0$ and set $w=\left(u_{j} \cdot v_{1}\right) v_{1}+\cdots+\left(u_{j} \cdot\right.$ $\left.v_{j-1}\right) v_{j-1}$. Since $v_{j}^{\prime} \cdot u_{k}=v_{j}^{\prime} \cdot v_{k}=0$ for any $k=1, \cdots, j-1$ and since $u_{j}=w+v_{j}^{\prime}$, we obtain

$$
Q\left(x_{1} u_{1}+\cdots+x_{j} u_{j}\right)=Q\left(x_{1} u_{1}+\cdots+x_{j-1} u_{j-1}+x_{j} w\right)
$$

for any $x_{1}, \cdots, x_{j} \in \mathbf{R}$. Therefore, according to the assumption, $\left\{u_{1}, \cdots, u_{j-1}, w\right\}$ is linearly independent over $\mathbf{R}$ with respect to $Q$. Moreover, Proposition 5 (i) implies that $\left\{u_{1}, \cdots, u_{j-1}, w\right\}$ is linearly independent over $\mathbf{C}$; however, this is impossible because $\left\{u_{1}, \cdots, u_{j-1}, w\right\} \subset \mathbf{C} v_{1}+\cdots+\mathbf{C} v_{j-1}$. Thus, we infer that $Q\left(v_{j}^{\prime}\right) \neq 0$ for any $j=3, \cdots, n$.

Q.E.D.

Corollary 2. Let $p \in \mathbf{N}, n=1, \cdots, p$ and $\left\{u_{1}, \cdots, u_{n}\right\} \subset \mathbf{C}^{p+1}$ be linearly independent over $\mathbf{R}$ with respect to $Q$. When $n=2$, further suppose $\operatorname{sgn}\left(u_{1}, u_{2}\right)=0$. Then there exists vectors $u_{n+1}, \cdots, u_{p+1} \in \mathbf{C}^{p+1}$ such that $\left\{u_{1}, \cdots, u_{p+1}\right\}$ is linearly independent over $\mathbf{R}$ with respect to $Q$. When $p=1$, it further follows that $\operatorname{sgn}\left(u_{1}, u_{2}\right)=0$. Moreover, if $\left\{u_{1}, \cdots, u_{n}\right\}$ is orthonormal with respect to $z \cdot w$, then $\left\{u_{1}, \cdots, u_{p+1}\right\}$ can be taken to be orthonormal.

REMARK. When $p=0$, set $u_{2}=i u_{1}$ for $u_{1} \neq 0$; then $\left\{u_{1}, u_{2}\right\}$ is linearly independent over $\mathbf{R}$ with respect to $Q$ with $\operatorname{sgn}\left(u_{1}, u_{2}\right) \neq 0$.

Proof. According to Proposition 6, there is an orthonormal system $\left\{v_{1}, \cdots\right.$, $\left.v_{n}\right\} \subset \mathbf{C} u_{1}+\cdots+\mathbf{C} u_{n}$ with respect to $z \cdot w$. If $\left\{u_{1}, \cdots, u_{n}\right\}$ is orthonormal, set $v_{j}=u_{j}$ for any $j=1, \cdots, n$. Then there exists a matrix $A \in S O(p+1, \mathrm{C})$ such that $A v_{j}=\mathbf{e}_{j}$ for $j=1, \cdots, n$. Set $v_{j}=A^{-1} \mathbf{e}_{j}$ for $j=n+1, \cdots, p+1$; then $\left\{v_{1}, \cdots, v_{p+1}\right\}$ is orthonormal with $u_{j} \cdot v_{k}=0$ for $j=1, \cdots, n, k=n+1, \cdots, p+1$. Further, set $U=\mathbf{R} u_{1}+\cdots+\mathbf{R} u_{n}$ and $u_{j}=\lambda v_{j}$ for $j=n+1, \cdots, p+1$, where $\lambda \in \mathbf{C}$ satisfies $|\lambda|=1$ and $\lambda^{2} \in Q(U)$. If $\left\{u_{1}, \cdots, u_{n}\right\}$ 
is orthonormal, we may set $\lambda=1$; then $\left\{u_{1}, \cdots, u_{p+1}\right\}$ is orthonormal.

If we set, for $x \in \mathbf{R}^{p+1}, F(x)=Q\left(x_{1} u_{1}+\cdots+x_{p+1} u_{p+1}\right)$; then we obtain

$$
F(x)=Q\left(x_{1} u_{1}+\cdots+x_{n} u_{n}\right)+\lambda^{2}\left(x_{n+1}^{2}+\cdots+x_{p+1}^{2}\right) .
$$

Therefore, according to the assumption and Proposition 4 (i) or (ii), we find that $F(x) \in Q(U) \backslash\{0\}$ for $x \neq 0$. Thus, $\left\{u_{1}, \cdots, u_{p+1}\right\}$ is linearly independent over $\mathbf{R}$ with respect to $Q$; if $p=1$, then we obtain $\operatorname{sgn}\left(u_{1}, u_{2}\right)=0$.

Q.E.D.

\section{Spaces of non-degenerate linear spaces.}

In this section we investigate non-degenerate oriented $\mathbf{R}$-linear subspaces of $\mathbf{C}^{p+1}$ in the real Grassmannians $\mathscr{G}_{n}\left(\mathbf{C}^{p+1}\right)$.

Definition 4. Let $p \in \mathbf{N}_{0}, n=1, \cdots, p+1$ and $U$ be an $n$-dimensional $\mathbf{R}$-linear subspace of $\mathbf{C}^{p+1}$. If $U \cap \Lambda_{0}=\{0\}$, then $U$ is said to be non-degenerate with respect to $Q$, or, shortly, non-degenerate.

By $\mathscr{G}_{n}\left(C^{p+1} \backslash \Lambda_{0}\right), n=1, \cdots, p+1$, we denote the set of all $n$-dimensional nondegenerate oriented $\mathbf{R}$-linear subspaces of $\mathbf{C}^{p+1}$; then $\mathscr{G}_{n}\left(\mathbf{C}^{p+1} \backslash \Lambda_{0}\right)$ is open in $\mathscr{G}_{n}\left(\mathbf{C}^{p+1}\right)$.

From Definitions 2 and 4 we find the following.

Proposition 7. Let $p \in \mathbf{N}_{0}, n=1, \cdots, p+1$. If $U \in \mathscr{G}_{n}\left(\mathbf{C}^{p+1} \backslash \Lambda_{0}\right)$, then a basis $\left\{u_{1}, \cdots, u_{n}\right\}$ for $U$ is linearly independent over $\mathbf{R}$ with respect to $Q$. Conversely, if $\left\{u_{1}, \cdots, u_{n}\right\} \subset \mathbf{C}^{p+1}$ is linearly independent over $\mathbf{R}$ with respect to $Q$, then $U=$ $\mathbf{R} u_{1}+\cdots+\mathbf{R} u_{n} \in \mathscr{G}_{n}\left(\mathbf{C}^{p+1} \backslash \Lambda_{0}\right)$.

By this proposition and Definition 3 we obtain the following.

Proposition 8. Let $p \in \mathbf{N}_{0}$; then $\mathscr{G}_{2}\left(\mathbf{C}^{p+1} \backslash \Lambda_{0}\right)$ is divided into three disjoint open subsets such that

$$
\mathscr{G}_{2}\left(\mathbf{C}^{p+1} \backslash \Lambda_{0}\right)=\mathscr{G}_{2}^{0}\left(\mathbf{C}^{p+1} \backslash \Lambda_{0}\right) \cup \mathscr{G}_{2}^{2+}\left(\mathbf{C}^{p+1} \backslash \Lambda_{0}\right) \cup \mathscr{G}_{2}^{2-}\left(\mathbf{C}^{p+1} \backslash \Lambda_{0}\right),
$$

where, for a basis $\left\{u_{1}, u_{2}\right\}$ for $U \in \mathscr{G}_{2}\left(\mathbf{C}^{p+1} \backslash \Lambda_{0}\right)$, we have

$$
U \in \mathscr{G}_{2}^{2 \lambda}\left(\mathbf{C}^{p+1} \backslash \Lambda_{0}\right) \Leftrightarrow \operatorname{sgn}\left(u_{1}, u_{2}\right)=\lambda .
$$

The set $\left\{\mathbf{e}_{j}, i \mathbf{e}_{j}\right\}$ is chosen for a basis for $\mathbf{C e}_{j}, j=0, \cdots, p$; then $\mathbf{C e}_{j} \in \mathscr{G}_{2}^{2+}\left(\mathbf{C}^{p+1} \backslash \Lambda_{0}\right)$ and $-\mathbf{C e}_{j} \in \mathscr{G}_{2}^{2-}\left(\mathbf{C}^{p+1} \backslash \Lambda_{0}\right)$.

The following is equivalent to Proposition 4.

PROPOSITION 9. Let $p \in \mathbf{N}_{0}, n=1, \cdots, p+1$.

(i) If $U \in \mathscr{G}_{n}\left(\mathbf{C}^{p+1} \backslash \Lambda_{0}\right)$ with $n \neq 2$, then $L=Q(U)$ is a closed convex cone in $\mathbf{C}$ such that $L \cap(-L)=\{0\}$.

(ii) If $U \in \mathscr{G}_{2}^{0}\left(\mathbf{C}^{p+1} \backslash \Lambda_{0}\right)$, then $L=Q(U)$ is a closed convex cone in $\mathbf{C}$ such that $L \cap(-L)=\{0\}$. 
(iii) If $U \in \mathscr{G}_{2}^{2+}\left(\mathbf{C}^{p+1} \backslash \Lambda_{0}\right) \cup \mathscr{G}_{2}^{2-}\left(\mathbf{C}^{p+1} \backslash \Lambda_{0}\right)$, then $Q(U)=\mathbf{C}$.

If $U \in \mathscr{G}_{n}\left(C^{p+1} \backslash \Lambda_{0}\right)$ with $n \neq 2$, or if $U \in \mathscr{G}_{2}^{0}\left(C^{p+1} \backslash \Lambda_{0}\right)$, then, by Proposition 9 (i) or (ii), $\langle z\rangle$ has two single-valued branches on $U$. When $n=1,\langle z\rangle$ has four branches on $U$ such that $\langle x u\rangle=|x|\langle u\rangle$ and $\langle x u\rangle=x\langle u\rangle$ for $u \in U \backslash\{0\}$ and $x \in \mathbf{R}$. If $U \in \mathscr{G}_{2}^{2+}\left(C^{p+1} \backslash \Lambda_{0}\right) \cup \mathscr{G}_{2}^{2-}\left(C^{p+1} \backslash \Lambda_{0}\right)$, then, by Definition 3 and Proposition 9 (iii), $\langle z\rangle$ has two single-valued branches on $U$.

By $\mathscr{G}_{n}\left(V_{0}^{p+1} \backslash \Lambda_{0}\right), n=1, \cdots, p+1$, we denote the set of all $n$-dimensional nondegenerate oriented R-linear subspaces of $V_{0}^{p+1}$. By Definition $3 \mathscr{G}_{2}\left(V_{0}^{p+1} \backslash \Lambda_{0}\right)$ is also divided into three disjoint open subsets like $\mathscr{G}_{2}\left(\mathbf{C}^{p+1} \backslash \Lambda_{0}\right)$ such that

$$
\mathscr{G}_{2}\left(V_{0}^{p+1} \backslash \Lambda_{0}\right)=\mathscr{G}_{2}^{0}\left(V_{0}^{p+1} \backslash \Lambda_{0}\right) \cup \mathscr{G}_{2}^{2+}\left(V_{0}^{p+1} \backslash \Lambda_{0}\right) \cup \mathscr{G}_{2}^{2-}\left(V_{0}^{p+1} \backslash \Lambda_{0}\right),
$$

where, for a basis $\left\{u_{1}, u_{2}\right\}$ for $U \in \mathscr{G}_{2}\left(V_{0}^{p+1} \backslash \Lambda_{0}\right)$, we have

$$
U \in \mathscr{G}_{2}^{2 \lambda}\left(V_{0}^{p+1} \backslash \Lambda_{0}\right) \Leftrightarrow \operatorname{sgn}\left(u_{1}, u_{2}\right)=\lambda \text {. }
$$

Proposition 10. Let $p \in \mathbf{N}_{0}, n=1, \cdots, p+1$ and $U \in \mathscr{G}_{n}\left(V_{0}^{p+1} \backslash \Lambda_{0}\right)$ for $n \neq 2$ (resp. $\left.U \in \mathscr{G}_{2}^{0}\left(V_{0}^{p+1} \backslash \Lambda_{0}\right)\right)$; then there exist an orthonormal system $\left\{v_{1}, \cdots, v_{m}\right\} \subset \mathbf{C}^{p+1}$ with respect to $z \cdot w$ and $\theta \in \mathbf{R}$ with $e^{i \theta} \in\langle U\rangle$ such that $U$ is joined to $\mathbf{R} e^{i \theta} v_{1}+\cdots+\mathbf{R} e^{i \theta} v_{n}$ in $\mathscr{G}_{n}\left(V_{0}^{p+1} \backslash \Lambda_{0}\right)$ for $n \neq 2$ (resp. $\mathscr{G}_{2}^{0}\left(V_{0}^{p+1} \backslash \Lambda_{0}\right)$ ) by an arc.

Proof. Let $\left\{u_{1}, \cdots, u_{n}\right\}$ be a basis for $U$ with $\left|\left\langle u_{1}\right\rangle\right|=\cdots=\left|\left\langle u_{n}\right\rangle\right|=1$. According to the assumption and Propositions 6, 7, 8, we construct an orthonormal system $\left\{v_{1}, \cdots, v_{n}\right\}$ from $\left\{u_{1}, \cdots, u_{n}\right\}$. Then we have $\left\langle v_{j}^{\prime}\right\rangle=u_{j} \cdot v_{j}$ for $j=1, \cdots, n$, and

$$
\left(u_{1} \cdots u_{n}\right)=\left(v_{1} \cdots v_{n}\right)\left(\begin{array}{ccc}
u_{1} \cdot v_{1} & \cdots & u_{n} \cdot v_{1} \\
& \ddots & \vdots \\
0 & & u_{n} \cdot v_{n}
\end{array}\right) .
$$

We define an arc $A_{n}:[0,1] \rightarrow \operatorname{Mat}(n, n, C)$ as

$$
A_{n}=\left(\begin{array}{cccc}
u_{1} \cdot v_{1} & \cdots & u_{n-1} \cdot v_{1} & u_{n} \cdot v_{1} \cos (\pi / 2) t \\
& \ddots & \vdots & \vdots \\
& & u_{n-1} \cdot v_{n-1} & u_{n} \cdot v_{n-1} \cos (\pi / 2) t \\
0 & & 0 & g_{n}(t)
\end{array}\right),
$$

where $g_{n}(t)=\sqrt{Q\left(u_{n}\right) \sin ^{2}(\pi / 2) t+\left(u_{n} \cdot v_{n}\right)^{2} \cos ^{2}(\pi / 2) t}$ with $g_{n}(1)=\left\langle u_{n}\right\rangle$. For $x \in \mathbf{R}^{n}$ we set $u^{\prime}=x_{1} u_{1}+\cdots+x_{n-1} u_{n-1}$ and $u_{n}(t)=\left(u_{n} \cdot v_{1}\right) \cos (\pi / 2) t v_{1}+\cdots+\left(u_{n} \cdot v_{n-1}\right) \cos (\pi / 2) t v_{n-1}+$ $g_{n}(t) v_{n}$; then we obtain

$$
\begin{aligned}
F_{t}(x)= & Q\left(\left(v_{1} \cdots v_{n}\right) A_{n}(t) x\right)=Q\left(u^{\prime}+x_{n} u_{n}(t)\right) \\
& =Q\left(u^{\prime}\right)+2 x_{n}\left(u^{\prime} \cdot u_{n}(t)\right)+x_{n}^{2} Q\left(u_{n}(t)\right) \\
& =Q\left(u^{\prime}\right)+2 \cos (\pi / 2) t x_{n}\left(u^{\prime} \cdot u_{n}\right)+x_{n}^{2} Q\left(u_{n}\right) \\
& =\cos (\pi / 2) t F_{0}(x)+(1-\cos (\pi / 2) t) F_{1}(x)
\end{aligned}
$$


The assumption and Proposition 9 (i), (ii) imply that $Q(U)$ is a convex cone with its vertex at 0 ; therefore, we have $F_{t}(x) \in Q(U) \backslash\{0\}$ for any $x \neq 0$ and $t \in[0,1]$.

By Proposition 7 we infer that $\left\{u_{1}, \cdots, u_{n-1}, u_{n}(t)\right\}$ is linearly independent over $\mathbf{R}$ with respect to $Q$; further, by Propositon 5 it is also linearly independent over $\mathbf{C}$. Hence, we find that $A_{n}(t) \in G L(n, \mathbf{C})$ and $g_{n}(t) \neq 0$ for any $t \in[0,1]$. Since $\left(u_{n} \cdot v_{n}\right)^{2}=Q\left(v_{n}^{\prime}\right)$, the value of $\left\langle v_{n}^{\prime}\right\rangle$ and $v_{n}$ are uniquely determined as $g_{n}(1)=\left\langle u_{n}\right\rangle$ by $g_{n}$; thus, we obtain

$$
A_{n}(1)=\left(\begin{array}{cccc}
u_{1} \cdot v_{1} & \cdots & u_{n-1} \cdot v_{1} & 0 \\
& \ddots & \vdots & \vdots \\
& & u_{n-1} \cdot v_{n-1} & 0 \\
0 & & 0 & \left\langle u_{n}\right\rangle
\end{array}\right) .
$$

On the same line of argument we construct the arc $A_{j}$ for $j=2, \cdots, n-1$ by replacing the $j$-th column of $A_{j+1}(1)$ with

$$
{ }^{t}\left(u_{j} \cdot v_{1} \cos (\pi / 2) t, \cdots, u_{j} \cdot v_{j-1} \cos (\pi / 2) t, g_{j}(t), 0, \cdots, 0\right),
$$

where $g_{j}(t)=\sqrt{Q\left(u_{j}\right) \sin ^{2}(\pi / 2) t+\left(u_{j} \cdot v_{j}\right)^{2} \cos ^{2}(\pi / 2) t}$; then each $v_{j}$ is uniquely determined by $g_{j}$. Thus, set $A=A_{n} \cup \cdots \cup A_{2}$; then $U$ and $\mathbf{R}\left\langle u_{1}\right\rangle v_{1}+\cdots+\mathbf{R}\left\langle u_{n}\right\rangle v_{n}$ are joined by $\left(v_{1} \cdots v_{n}\right) A \mathbf{R}^{n}$. From the assumption it follows that $\left\langle u_{j}\right\rangle=e^{i \theta_{j}}$ for $j=1, \cdots, n$, where $\left|\theta_{j}-\theta_{k}\right|<\pi / 2$. We define $V:[0,1] \rightarrow \mathscr{G}_{n}\left(V_{0}^{p+1} \backslash \Lambda_{0}\right)$ as

$$
V(t)=\mathbf{R} e^{i \theta_{1}} v_{1}+\mathbf{R} e^{i \theta_{1}+i\left(\theta_{2}-\theta_{1}\right)(1-t)} v_{2}+\cdots+\mathbf{R} e^{i \theta_{1}+i\left(\theta_{n}-\theta_{1}\right)(1-t)} v_{n} ;
$$

then $V(1)=\mathbf{R} e^{i \theta_{1}} v_{1}+\cdots+\mathbf{R} e^{i \theta_{1}} v_{n}$.

Q.E.D.

Proposition 11. Let $p \in \mathbf{N}_{0}, n=1, \cdots, p+1, U \in \mathscr{G}_{n}\left(V_{0}^{p+1} \backslash \Lambda_{0}\right)$ for $n \neq 2$ (resp. $\left.U \in \mathscr{G}_{2}^{0}\left(V_{0}^{p+1} \backslash \Lambda_{0}\right)\right)$ and $\left.\langle z\rangle\right|_{ \pm \mathbf{R}^{n}}=|z|$ for $n \geqq 2$ while $\left.\langle z\rangle\right|_{ \pm \mathbf{R}}=|z|$ or $\left.\langle z\rangle\right|_{ \pm \mathbf{R}}=z$ for $n=1$; then $U$ is joined to $\pm \mathbf{R}^{p+1}$ in $\mathscr{G}_{n}\left(V_{0}^{p+1} \backslash \Lambda_{0}\right)$ for $n \neq 2\left(\right.$ resp. $\left.\mathscr{G}_{2}^{0}\left(V_{0}^{p+1} \backslash \Lambda_{0}\right)\right)$ by an arc. If $n \leqq p$, then $U$ is joined to $\mathbf{R}^{n}$ by an arc.

Proof. According to Proposition 10, there are an orthonormal system $\left\{v_{1}, \cdots, v_{n}\right\}$ and $\theta \in \mathbf{R}$ with $e^{i \theta} \in\langle U\rangle$ such that $U$ is joined to $\mathbf{R} e^{i \theta} v_{1}+\cdots+\mathbf{R} e^{i \theta} v_{n}$ in $\mathscr{G}_{n}\left(V_{0}^{p+1} \backslash \Lambda_{0}\right)$ for $n \neq 2$ (resp. $\left.\mathscr{G}_{2}^{0}\left(V_{0}^{p+1} \backslash \Lambda_{0}\right)\right)$ by an arc. We set $V(t)=\mathbf{R} e^{i \theta(1-t)} v_{1}+\cdots+\mathbf{R} e^{i \theta(1-t)} v_{n}$; then the arc $V:[0,1] \rightarrow \mathscr{G}_{n}\left(V_{0}^{p+1} \backslash \Lambda_{0}\right)$ for $n \neq 2\left(\right.$ resp. $\left.V:[0,1] \rightarrow \mathscr{G}_{2}^{0}\left(V_{0}^{p+1} \backslash \Lambda_{0}\right)\right)$ satisfies $V(1)=\mathbf{R} v_{1}+\cdots+\mathbf{R} v_{n}$. Since the $(p+1, n)$-matrix $\left(v_{1} \cdots v_{n}\right)$ is joined to $\left(\mathbf{e}_{0} \cdots \mathbf{e}_{n-1}\right)$ for $n \leqq p$ and to $\left(\mathbf{e}_{0} \cdots \pm \mathbf{e}_{p}\right)$ for $n=p+1$ by an $\operatorname{arc} A:[0,1] \rightarrow \operatorname{Mat}(p+1, n, \mathbf{C})$ such that the columns of $A(t)$ are orthonormal, we obtain the result.

Q.E.D.

Proposition 12. Let $p \in \mathbf{N}_{0}, U \in \mathscr{G}_{2}^{2+}\left(V_{0}^{p+1} \backslash \Lambda_{0}\right)$ (resp. $\left.U \in \mathscr{G}_{2}^{2-}\left(V_{0}^{p+1} \backslash \Lambda_{0}\right)\right)$ and $\left.\langle z\rangle\right|_{ \pm \mathbf{C e}_{0}}=z$; then $U$ is joined to $\mathbf{C e}_{0}$ in $\mathscr{G}_{2}^{2+}\left(V_{0}^{p+1} \backslash \Lambda_{0}\right)\left(\right.$ resp. $-\mathbf{C e}_{0}$ in $\left.\mathscr{G}_{2}^{2-}\left(V_{0}^{p+1} \backslash \Lambda_{0}\right)\right)$ by an arc.

Proof. We may suppose that $U \in \mathscr{G}_{2}^{2+}\left(V_{0}^{p+1} \backslash \Lambda_{0}\right)$. Since we have $\langle U\rangle=\mathbf{C}$ by Definition 3 and Proposition 9 (iii), there are $u_{1}, u_{2} \in U$ such that $\left\langle u_{1}\right\rangle=1$ and $\left\langle u_{2}\right\rangle=i$; then $\left\{u_{1}, u_{2}\right\}$ is linearly independent over $\mathbf{R}$. From Proposition 7 it follows that $\left\{u_{1}, u_{2}\right\}$ 
is linearly independent over $\mathbf{R}$ with respect to $Q$. Therefore, $\operatorname{since} \operatorname{sgn}\left(u_{1}, u_{2}\right)=1,\left\{u_{1}, u_{2}\right\}$ is a basis for $U$. Furthermore, we obtain

$$
Q\left(u_{1} \cos \theta+u_{2} \sin \theta\right)=\cos 2 \theta+\left(u_{1} \cdot u_{2}\right) \sin 2 \theta ;
$$

then since $U \in \mathscr{G}_{2}^{2+}\left(V_{0}^{p+1} \backslash \Lambda_{0}\right)$, we have $\operatorname{Im}\left(u_{1} \cdot u_{2}\right)>0$. Set $v=u_{2}-\left(u_{1} \cdot u_{2}\right) u_{1}$; then we have $u_{1} \cdot v=0, Q(v)=-1-\left(u_{1} \cdot u_{2}\right)^{2}$ and moreover,

$$
\left(u_{1} u_{2}\right)=\left(u_{1} v\right)\left(\begin{array}{cc}
1 & u_{1} \cdot u_{2} \\
0 & 1
\end{array}\right) \text {. }
$$

We define an $\operatorname{arc} A:[0,1] \rightarrow \operatorname{Mat}(2,2, \mathrm{C})$ as

$$
A(t)=\left(\begin{array}{cc}
1 & g(t) \\
& \cos (\pi / 2) t
\end{array}\right)
$$

where $g(t)=\sqrt{\left(u_{1} \cdot u_{2}\right)^{2} \cos ^{2}(\pi / 2) t-\sin ^{2}(\pi / 2) t}$. Since $\operatorname{Im}\left(u_{1} \cdot u_{2}\right)>0$, we have $\left(u_{1} \cdot u_{2}\right)^{2} \in$ $\mathbf{C} \backslash \mathbf{R}_{+}$and $g(t)^{2} \in \mathbf{C} \backslash \mathbf{R}_{+}$; therefore, the value of $g(t)$ is uniquely determined as $g(0)=u_{1} \cdot u_{2}$. Then we obtain $\operatorname{Im} g(t)>0$ with $g(1)=i$ and furthermore, $A(1)=\left(\begin{array}{ll}1 & i \\ 0 & 0\end{array}\right)$. Thus, we find for any $\theta \in[0,2 \pi]$

$$
\begin{aligned}
F_{t}(\theta) & =Q\left(\left(u_{1} v\right) A(t)\left(\begin{array}{c}
\cos \theta \\
\sin \theta
\end{array}\right)\right) \\
& =\cos ^{2} \theta+2 g(t) \cos \theta \sin \theta+\left(g(t)^{2}+\cos ^{2}(\pi / 2) t Q(v)\right) \sin ^{2} \theta \\
& =\cos 2 \theta+g(t) \sin 2 \theta .
\end{aligned}
$$

Since $\operatorname{Im} g(t)>0$, we infer that $\operatorname{sgn}\left(\left(u_{1} v\right) A(t)\right)=1$; therefore, $U$ is joined to $\mathrm{C} u_{1}$ in $\mathscr{G}_{2}^{2+}\left(V_{0}^{p+1} \backslash \Lambda_{0}\right)$ by an arc. Moreover, since $u_{1}$ is joined to $e_{0}$ by an arc $w:[0,1] \rightarrow$ $V_{0}^{p+1} \backslash \Lambda_{0}$ such that $\langle w(t)\rangle=1$, we obtain an arc joining $U$ and $\mathbf{C e}_{0}$ in $\mathscr{G}_{2}^{2+}\left(V_{0}^{p+1} \backslash \Lambda_{0}\right)$.

Q.E.D.

THEOREM 1. Let $p \in \mathbf{N}_{0}$; then $\mathscr{G}_{p+1}\left(\mathbf{C}^{p+1} \backslash \Lambda_{0}\right)$ and $\mathscr{G}_{p+1}\left(V_{0}^{p+1} \backslash \Lambda_{0}\right)$ are divided into such domains as follows.

(i) If $p(\geqq 0)$ is even, then $\mathscr{G}_{p+1}\left(\mathbf{C}^{p+1} \backslash \Lambda_{0}\right)$ is connected.

(ii) If $p=1$, then

$$
\mathscr{G}_{2}\left(\mathbf{C}^{2} \backslash \Lambda_{0}\right)=\mathscr{G}_{2}^{2+}\left(\mathbf{C}^{2} \backslash \Lambda_{0}\right) \cup \mathscr{G}_{2}^{+}\left(\mathbf{C}^{2} \backslash \Lambda_{0}\right) \cup \mathscr{G}_{2}^{-}\left(\mathbf{C}^{2} \backslash \Lambda_{0}\right) \cup \mathscr{G}_{2}^{2-}\left(\mathbf{C}^{2} \backslash \Lambda_{0}\right),
$$

where $\mathscr{G}_{2}^{0}\left(\mathbf{C}^{2} \backslash \Lambda_{0}\right)=\mathscr{G}_{2}^{+}\left(\mathbf{C}^{2} \backslash \Lambda_{0}\right) \cup \mathscr{G}_{2}^{-}\left(\mathbf{C}^{2} \backslash \Lambda_{0}\right)$ with $\pm \mathbf{R}^{2} \in \mathscr{G}_{2}^{ \pm}\left(\mathbf{C}^{2} \backslash \Lambda_{0}\right)$ and $\pm \mathbf{C} \mathbf{e}_{0} \in$ $\mathscr{G}_{2}^{2 \pm}\left(\mathbf{C}^{2} \backslash \Lambda_{0}\right)$.

(iii) If $p(\geqq 3)$ is odd, then

$$
\mathscr{G}_{p+1}\left(\mathbf{C}^{p+1} \backslash \Lambda_{0}\right)=\mathscr{G}_{p+1}^{+}\left(\mathbf{C}^{p+1} \backslash \Lambda_{0}\right) \cup \mathscr{G}_{p+1}^{-}\left(\mathbf{C}^{p+1} \backslash \Lambda_{0}\right),
$$


where $\pm \mathbf{R}^{p+1} \in \mathscr{G}_{p+1}^{ \pm}\left(\mathbf{C}^{p+1} \backslash \Lambda_{0}\right)$.

(iv) If $p=0$, then

$$
\mathscr{G}_{1}\left(V_{0}^{1} \backslash \Lambda_{0}\right)=\mathscr{G}_{1}^{2+}\left(V_{0}^{1} \backslash \Lambda_{0}\right) \cup \mathscr{G}_{1}^{+}\left(V_{0}^{1} \backslash \Lambda_{0}\right) \cup \mathscr{G}_{1}^{-}\left(V_{0}^{1} \backslash \Lambda_{0}\right) \cup \mathscr{G}_{1}^{2-}\left(V_{0}^{1} \backslash \Lambda_{0}\right),
$$

where $\pm \mathbf{R} \in \mathscr{G}_{1}^{ \pm}\left(V_{0}^{1} \backslash \Lambda_{0}\right)$ for $\left.\langle z\rangle\right|_{ \pm \mathbf{R}}=|z|$ and $\pm \mathbf{R} \in \mathscr{G}_{1}^{2 \pm}\left(V_{0}^{1} \backslash \Lambda_{0}\right)$ for $\left.\langle z\rangle\right|_{ \pm \mathbf{R}}=z$.

(v) If $p=1$, then

$$
\mathscr{G}_{2}\left(V_{0}^{2} \backslash \Lambda_{0}\right)=\mathscr{G}_{2}^{2+}\left(V_{0}^{2} \backslash \Lambda_{0}\right) \cup \mathscr{G}_{2}^{+}\left(V_{0}^{2} \backslash \Lambda_{0}\right) \cup \mathscr{G}_{2}^{-}\left(V_{0}^{2} \backslash \Lambda_{0}\right) \cup \mathscr{G}_{2}^{2-}\left(V_{0}^{2} \backslash \Lambda_{0}\right),
$$

where $\pm \mathbf{R}^{2} \in \mathscr{G}_{2}^{ \pm}\left(V_{0}^{2} \backslash \Lambda_{0}\right)$ for $\left.\langle z\rangle\right|_{ \pm \mathbf{R}^{2}}=|z|$ and $\pm \mathbf{C e}_{0} \in \mathscr{G}_{2}^{2 \pm}\left(V_{0}^{2} \backslash \Lambda_{0}\right)$ for $\left.\langle z\rangle\right|_{ \pm \mathbf{C e}_{0}}=z$.

(vi) If $p \geqq 2$, then

$$
\mathscr{G}_{p+1}\left(V_{0}^{p+1} \backslash \Lambda_{0}\right)=\mathscr{G}_{p+1}^{+}\left(V_{0}^{p+1} \backslash \Lambda_{0}\right) \cup \mathscr{G}_{p+1}^{-}\left(V_{0}^{p+1} \backslash \Lambda_{0}\right),
$$

where $\pm \mathbf{R}^{p+1} \in \mathscr{G}_{p+1}^{ \pm}\left(V_{0}^{p+1} \backslash \Lambda_{0}\right)$ for $\left.\langle z\rangle\right|_{ \pm \mathbf{R}^{p+1}}=|z|$.

Proof. Let $U:[0,1] \rightarrow \mathscr{G}_{p+1}\left(V_{0}^{p+1} \backslash \Lambda_{0}\right)$ for $p \neq 1$ (resp. $U:[0,1] \rightarrow \mathscr{G}_{2}^{0}\left(V_{0}^{2} \backslash \Lambda_{0}\right)$ ) be an arc such that $U(0)=\mathbf{R}^{p+1}$ and $U(1)= \pm \mathbf{R}^{p+1}$. Furthermore, let $\left\{u_{0}(t), \cdots, u_{p}(t)\right\}$ be a basis for $U(t)$ such that each $u_{j}:[0,1] \rightarrow V_{0}^{p+1} \backslash \Lambda_{0}$ is continuous with $u_{j}(0)=\mathbf{e}_{j}, u_{j}(1)= \pm \mathbf{e}_{j}$ for $j=0, \cdots, p$. According to Proposition 10, there exist an orthonormal system $\left\{v_{0}(t), \cdots, v_{p}(t)\right\}$ and $\theta(t) \in \mathbf{R}$ with $e^{i \theta(t)} \in\langle U(t)\rangle$ such that $U$ is joined to $V=\mathbf{R} e^{i \theta} v_{0}+\cdots+\mathbf{R} e^{i \theta} v_{p}$ in $\mathscr{G}_{p+1}\left(V_{0}^{p+1} \backslash \Lambda_{0}\right)$ for $p \neq 1$ (resp. $\left.\mathscr{G}_{2}^{0}\left(V_{0}^{2} \backslash \Lambda_{0}\right)\right)$ by an arc. By the assumption and Proposition 6 we find that $u_{j}(0)=v_{j}(0)$ and $u_{j}(1)=\left\langle u_{j}(1)\right\rangle v_{j}(1)$ for $j=0, \cdots, p$. On the other hand, since $U(1)= \pm \mathbf{R}^{p+1}$, we have $\left\langle u_{0}(1)\right\rangle=\cdots=\left\langle u_{p}(1)\right\rangle=$ \pm 1 . Therefore, since $\left(v_{0}(t) \cdots v_{p}(t)\right) \in S O(p+1, \mathrm{C})$, we infer that

$$
\operatorname{det}\left(u_{0}(1) \cdots u_{p}(1)\right)=\left\langle u_{0}(1)\right\rangle \cdots\left\langle u_{p}(1)\right\rangle=( \pm 1)^{p+1} .
$$

Then $U(1)=\mathbf{R}^{p+1}$ for odd $p \geqq 0$ and $U(1)=\left\langle u_{j}(1)\right\rangle \mathbf{R}^{p+1}$ for even $p \geqq 0$. Therefore, Proposition 11 implies (iii), (iv), (vi). Since, for even $p \geqq 0, \mathbf{R}^{p+1}$ and $-\mathbf{R}^{p+1}$ are joined in $\mathscr{G}_{p+1}\left(\mathbf{C}^{p+1} \backslash \Lambda_{0}\right)$ by an arc $U(t)=\mathbf{R} e^{i \pi t} \mathbf{e}_{0}+\cdots+\mathbf{R} e^{i \pi t} \mathbf{e}_{p}$, we have (i). Moreover, according to Propositions 8, 11, 12, we obtain (ii), (v). Q.E.D.

THEOREM 2. Let $p \in \mathbf{N}, n=1, \cdots, p$; then $\mathscr{G}_{n}\left(\mathbf{C}^{p+1} \backslash \Lambda_{0}\right)$ and $\mathscr{G}_{n}\left(V_{0}^{p+1} \backslash \Lambda_{0}\right)$ are divided into such domains as follows.

(i) If $n \neq 2$, then $\mathscr{G}_{n}\left(\mathbf{C}^{p+1} \backslash \Lambda_{0}\right)$ is connected.

(ii) If $n=2$, then

$$
\mathscr{G}_{2}\left(\mathbf{C}^{p+1} \backslash \Lambda_{0}\right)=\mathscr{G}_{2}^{2+}\left(\mathbf{C}^{p+1} \backslash \Lambda_{0}\right) \cup \mathscr{G}_{2}^{0}\left(\mathbf{C}^{p+1} \backslash \Lambda_{0}\right) \cup \mathscr{G}_{2}^{2-}\left(\mathbf{C}^{p+1} \backslash \Lambda_{0}\right),
$$

where $\pm \mathbf{C e}_{0} \in \mathscr{G}_{2}^{2 \pm}\left(\mathbf{C}^{p+1} \backslash \Lambda_{0}\right)$ and $\mathbf{R}^{2} \in \mathscr{G}_{2}^{0}\left(\mathbf{C}^{p+1} \backslash \Lambda_{0}\right)$.

(iii) If $n=1$, then

$$
\mathscr{G}_{1}\left(V_{0}^{p+1} \backslash \Lambda_{0}\right)=\mathscr{G}_{1}^{0}\left(V_{0}^{p+1} \backslash \Lambda_{0}\right) \cup \mathscr{G}_{1}^{00}\left(V_{0}^{p+1} \backslash \Lambda_{0}\right),
$$

where $\mathbf{R} \in \mathscr{G}_{1}^{0}\left(V_{0}^{p+1} \backslash \Lambda_{0}\right)$ for $\left.\langle z\rangle\right|_{\mathbf{R}}=|z|$ and $\mathbf{R} \in \mathscr{G}_{1}^{00}\left(V_{0}^{p+1} \backslash \Lambda_{0}\right)$ for $\left.\langle z\rangle\right|_{\mathbf{R}}=z$.

(iv) If $n=2$, then 


$$
\begin{gathered}
\mathscr{G}_{2}\left(V_{0}^{p+1} \backslash \Lambda_{0}\right)=\mathscr{G}_{2}^{2+}\left(V_{0}^{p+1} \backslash \Lambda_{0}\right) \cup \mathscr{G}_{2}^{0}\left(V_{0}^{p+1} \backslash \Lambda_{0}\right) \cup \mathscr{G}_{2}^{2-}\left(V_{0}^{p+1} \backslash \Lambda_{0}\right), \\
\text { where } \pm \mathbf{C e}_{0} \in \mathscr{G}_{2}^{2 \pm}\left(V_{0}^{p+1} \backslash \Lambda_{0}\right) \text { for }\left.\langle z\rangle\right|_{ \pm \mathbf{C e}_{0}}=z \text { and } \mathbf{R}^{2} \in \mathscr{G}_{2}^{0}\left(V_{0}^{p+1} \backslash \Lambda_{0}\right) \text { for }\left.\langle z\rangle\right|_{\mathbf{R}^{2}}=|z|
\end{gathered}
$$

(v) If $n \neq 2$, then $\mathscr{G}_{n}\left(V_{0}^{p+1} \backslash \Lambda_{0}\right)$ is connected.

Proof. Proposition 11 implies (i) and (v) while Propositions 8, 11 and 12 imply (ii) and (iv). Furthermore, we define a map $F$ as $F: V_{0}^{p+1} \backslash \Lambda_{0} \ni z \mapsto\langle-z\rangle \mid\langle z\rangle \in C$; then for $U \in \mathscr{G}_{1}\left(V_{0}^{p+1} \backslash \Lambda_{0}\right)$ we find $F(U \backslash\{0\})=\{1\},\{-1\}$. Therefore, according to Proposition 11, we obtain (iii).

Q.E.D.

Corollary 3. Let $p \in \mathbf{N}, n=2, \cdots, p+1, U \in \mathscr{G}_{n}\left(\mathbf{C}^{p+1} \backslash \Lambda_{0}\right)\left(\right.$ resp. $U \in \mathscr{G}_{n}\left(V_{0}^{p+1} \backslash\right.$ $\left.\Lambda_{0}\right)$ ) and $V$ be an $m$-dimensional $\mathbf{R}$-linear subspace of $U$, where $m=1, \cdots, n$.

(i) If $m \neq 2$, then $V \in \mathscr{G}_{m}\left(C^{p+1} \backslash \Lambda_{0}\right)\left(\right.$ resp. $\left.V \in \mathscr{G}_{m}\left(V_{0}^{p+1} \backslash \Lambda_{0}\right)\right)$.

(ii) If $m=2$, then $V \in \mathscr{G}_{2}^{0}\left(C^{p+1} \backslash \Lambda_{0}\right)\left(\right.$ resp. $\left.V \in \mathscr{G}_{2}^{0}\left(V_{0}^{p+1} \backslash \Lambda_{0}\right)\right)$.

Proof. From Propositions 1, 3 and 7 the assertion follows immediately.

Q.E.D.

\section{Cauchy's intergral formula on manifolds.}

In this section we consider Cauchy's integral formula for complex regular functions $f$ in real $(p+1)$-dimensional connected submanifolds $M$ of $\mathbf{C}^{p+1}$ with boundary.

Proposition 13. Let $p, q \in \mathbf{N}_{0}$ with $p \leqq q, n=p, p+1, \Omega$ be a domain of $\mathbf{C}^{p+1} \subset \mathbf{C}_{p}$, $f: \Omega \rightarrow \mathbf{C}_{q}$ be holomorphic and $M$ be a real n-dimensional smooth submanifold of $\Omega$ with $T_{a} M \in \mathscr{G}_{n}\left(C^{p+1} \backslash \Lambda_{0}\right)(n \neq 2), T_{a} M \in \mathscr{G}_{2}^{0}\left(C^{p+1} \backslash \Lambda_{0}\right)$ for some $a \in M$.

(i) If $n=p+1$, then $\left.D_{z} f\right|_{M}=0$ (resp. $\left.f D_{z}\right|_{M}=0$ ) implies that $f$ is complex left (resp. right) regular.

(ii) If $n=p$ and if $f$ is complex regular, then $\left.f\right|_{M}=0$ implies $f=0$.

ProOF. (i): According to the assumption, there exists an open neighborhood $U$ of $a$ in $M$ such that $T_{z} M \in \mathscr{G}_{p+1}\left(C^{p+1} \backslash \Lambda_{0}\right)(p \neq 1), T_{z} M \in \mathscr{G}_{2}^{0}\left(C^{2} \backslash \Lambda_{0}\right)$ for any $z \in U$. Since $D_{z} f$ is holomorphic on $\Omega$ with $\left.D_{z} f\right|_{M}=0$, by Propositions 5, 7 and 8 we obtain

$$
\left.\frac{\partial}{\partial z_{j}} D_{z} f\right|_{U}=0 \quad \text { for any } j=0, \cdots, p \text {. }
$$

In the same way it is proved that all the derivatives of $D_{z} f$ are zero in $M$; thus, since $D_{z} f$ is holomorphic, we obtain $D_{z} f=0$. On the same line of argument it is shown that $\left.f D_{z}\right|_{M}=0$ implies $f D_{z}=0$.

(ii): If $p=0$, then $f$ is constant on $\Omega$; therefore, the assertion is clearly true. According to the assumption, there exists an open neighborhood $U$ of $a$ in $M$ such that $T_{z} M \in \mathscr{G}_{p}\left(\mathbf{C}^{p+1} \backslash \Lambda_{0}\right)(p \neq 2), T_{z} M \in \mathscr{G}_{2}^{0}\left(\mathbf{C}^{3} \backslash \Lambda_{0}\right)$ for any $z \in U$. Let $\left\{u_{0}, \cdots, u_{p-1}\right\}$ be a basis for $T_{z} M$; then, from Propositions 7 and 8 and Corollary 2 it follows that there 
exists a vector $u_{p} \in \mathbf{C}^{p+1}$ such that $\left\{u_{0}, \cdots, u_{p}\right\}$ is linearly independent over $\mathbf{R}$ with respect to $Q$. When $p=1$, it further follows that $\operatorname{sgn}\left(u_{0}, u_{1}\right)=0$. If $U$ is sufficiently small, we can choose a common vector $u_{p} \in \mathbf{C}^{p+1}$ for any $z \in U$. By Proposition 6 , we construct an orthonormal system $\left\{v_{0}, \cdots, v_{p}\right\}$ from $\left\{u_{0}, \cdots, u_{p}\right\}$. Since, by Proposition 5, $\left\{u_{0}, \cdots, u_{p}\right\}$ and $\left\{v_{0}, \cdots, v_{p}\right\}$ are bases for $C^{p+1}$, let $\left(z_{0}^{\prime}, \cdots, z_{p}^{\prime}\right)$ and $\left(z_{0}^{\prime \prime}, \cdots, z_{p}^{\prime \prime}\right)$ be the coordinates of $z \in \mathbf{C}^{p+1}$ for $\left\{u_{0}, \cdots, u_{p}\right\}$ and $\left\{v_{0}, \cdots, v_{p}\right\}$ respectively. Then, we have

$$
\left(\begin{array}{c}
\left(\partial f / \partial z_{0}^{\prime}\right)(z) \\
\vdots \\
\left(\partial f / \partial z_{p}^{\prime}\right)(z)
\end{array}\right)=\left(\begin{array}{ccc}
u_{0} \cdot v_{0} & & 0 \\
\vdots & \ddots & \\
u_{p} \cdot v_{0} \cdots & u_{p} \cdot v_{p}
\end{array}\right)\left(\begin{array}{c}
\left(\partial f / \partial z_{0}^{\prime \prime}\right)(z) \\
\vdots \\
\left(\partial f / \partial z_{p}^{\prime \prime}\right)(z)
\end{array}\right) \quad \text { for any } z \in U
$$

Since $\left.f\right|_{M}=0$ by the assumption, we find that

$$
\frac{\partial f}{\partial z_{j}^{\prime}}(z)=0 \quad \text { for any } j=0, \cdots, p-1 \text { and } z \in U \text {; }
$$

therefore, since $u_{j} \cdot v_{j} \neq 0$ for any $j=0, \cdots, p-1$, we obtain

$$
\frac{\partial f}{\partial z_{j}^{\prime \prime}}(z)=0 \quad \text { for any } j=0, \cdots, p-1 \text { and } z \in U \text {. }
$$

Furthemore, we have

$$
\left(\begin{array}{c}
\left(\partial f / \partial z_{0}\right)(z) \\
\vdots \\
\left(\partial f / \partial z_{p}\right)(z)
\end{array}\right)=\left(v_{0} \cdots v_{p}\right)\left(\begin{array}{c}
\left(\partial f / \partial z_{0}^{\prime \prime}\right)(z) \\
\vdots \\
\left(\partial f / \partial z_{p}^{\prime \prime}\right)(z)
\end{array}\right)=v_{p} \frac{\partial f}{\partial z_{p}^{\prime \prime}}(z) \quad \text { for any } z \in U \text {; }
$$

hence, from the assumption $D_{z} f=0$ (resp. $f D_{z}=0$ ) we infer that $v_{p}\left(\partial f / \partial z_{p}^{\prime \prime}\right)(z)=0$ (resp. $\left.\left(\partial f / \partial z_{p}^{\prime \prime}\right)(z) v_{p}=0\right)$ for any $z \in U$, where we consider the vector $v_{p} \in \mathbf{C}^{p+1}$ as a Clifford number $v_{p} \in \mathbf{C}_{p}$. Since $\tilde{v}_{p} v_{p}=v_{p} \tilde{v}_{p}=Q\left(v_{p}\right)=1$, we obtain $\left(\partial f / \partial z_{p}^{\prime \prime}\right)(z)=0$ for any $z \in U$; therefore, we conclude that

$$
\partial f /\left.\partial z_{j}\right|_{U}=0 \quad \text { for any } j=0, \cdots, p .
$$

In the same way it is proved that all the derivatives of $f$ are zero in $U$; thus, since $f$ is holomorphic, we obtain $f=0$.

Q.E.D.

REMARK 1. Let $\Omega$ be a domain of $\mathbf{C}_{2}\left(\cong \mathbf{C}^{4}\right)$ and $f: \Omega \rightarrow \mathbf{C}_{q}(2 \leqq q)$ be holomorphic; then we obtain analogous results for $f$.

REMARK 2. Let $\Omega$ be a domain of $\mathbf{R}^{p+1}\left(\operatorname{resp} . \mathbf{R}_{2}\left(\cong \mathbf{R}^{4}\right)\right), M$ be a real $p$-dimensional (resp. 3-dimensional) smooth submanifold of $\Omega$ and $f: \Omega \rightarrow \mathbf{C}_{q}, p \leqq q$ (resp. $2 \leqq q$ ), be regular, or monogenic. Then in a way analogous to the proof of (ii) it is proved that $\left.f\right|_{M}=0$ implies $f=0 . \operatorname{In}[2, \S 10, \S 28]$ the same result is obtained by the Painleve theorem.

Corollary 4. Let $p, q \in \mathbf{N}_{0}$ with $p \leqq q, \Omega$ be a domain of $\mathbf{C}^{p+1}, f: \Omega \rightarrow \mathbf{C}_{q}$ be complex regular and $M$ be $a(p+1)$-dimensional smooth submanifold of $\Omega$ with 
$T_{a} M \in \mathscr{G}_{p+1}\left(C^{p+1} \backslash \Lambda_{0}\right)$ for some $a \in M$. Then $\left.f\right|_{M}=0$ implies $f=0$.

Proof. If $p=0$, then $f$ is constant on $\Omega$; therefore, the assertion is clearly true. According to Corollary 3, a real $p$-dimensional smooth submanifold $N(\ni a)$ of $M$ satisfies $T_{a} N \in \mathscr{G}_{p}\left(C^{p+1} \backslash \Lambda_{0}\right)$ for $p \neq 2$ or $T_{a} N \in \mathscr{G}_{2}^{0}\left(C^{3} \backslash \Lambda_{0}\right)$. Thus, by Proposition 13 (ii), $\left.f\right|_{N}=0$ implies $f=0$.

Q.E.D.

By Stokes' theorem we obtain the following Cauchy integral theorem immediately (cf. $[2, \S 9])$.

THEOREM 3 (Cauchy's Integral Theorem). Let $p, q \in \mathbf{N}_{0}$ with $p \leqq q, \Omega$ be a domain of $\mathbf{C}^{p+1}, f: \Omega \rightarrow \mathbf{C}_{q}$ be complex right regular, $g: \Omega \rightarrow \mathbf{C}_{q}$ be complex left regular, $d \sigma_{z}$ be the $\mathbf{C}_{p}$-valued surface-element (defined in $\left.\$ 2\right)$ and $M$ be a real $(p+1)$-dimensional piecewise smooth compact oriented submanifold of $\Omega$ with boundary. Then

$$
\int_{\partial M} f d \sigma_{z} g=0 \text {. }
$$

Let $\Omega$ be a domain of $\mathbf{C}^{p+1}, K$ be compact in $\Omega$ and $L$ be connected in $\Omega \backslash \Lambda(K)$. Then by $D_{\Omega}(K, L)$ we denote the domain of $\Omega \backslash \Lambda(K)$ which includes $L$. We set $B_{r}=\left\{z \in \mathbf{C}^{p+1}|| z \mid \leqq r\right\}$.

To begin with, we show Cauchy's integral formula for $p=1$.

THEOREM 4 (Cauchy's Integral Formula). Let $q \in \mathbf{N}, \lambda= \pm 1, \Omega$ be a domain of $\mathbf{C}^{2} \subset \mathbf{C}_{1}, f: \Omega \rightarrow \mathbf{C}_{q}$ be complex right regular, $a \in \Omega$ and $M$ be a real 2-dimensional piecewise smooth compact connected oriented submanifold of $\Omega$ with boundary such that $a \in M^{\circ}, M$ is smooth at $a, M \cap \Lambda_{a}=\{a\}$ and $T_{a} M \in \mathscr{G}_{2}^{2 \lambda}\left(C^{2} \backslash \Lambda_{0}\right)$. Then

$$
f(z)=\frac{\lambda}{2 \pi i} \int_{\partial M} f(w) \frac{d w}{w-z} \quad \text { for any } z \in D_{\Omega}(\partial M, a),
$$

where $1 /(w-z)$ is the inverse element of a Clifford number $w-z \in \mathbf{C}^{2} \subset \mathbf{C}_{1}$.

Proof. By $F_{z}(w)$ we denote the integrand in the right side of the formula. Since $M$ is smooth, for any $\varepsilon>0$ there exists an open neighborhood $U_{a}$ of $a$ in $M^{\circ}$ such that the projection $\phi_{a}: U_{a} \rightarrow a+T_{a} M$ is an into diffeomorphism with

$$
\left|w-\phi_{a}(w)\right| \leqq \varepsilon\left|\phi_{a}(w)-a\right| \quad \text { for any } w \in U_{a} .
$$

Then there is a number $\delta>0$ such that $a+B_{2 \delta} \subset \Omega$ and further, such that the following holds. Set $C_{\delta}=B_{\delta} \cap T_{a} M$; then $a+C_{\delta} \subset \phi_{a}\left(U_{a}\right)$. Set $D_{\delta}=\phi_{a}^{-1}\left(a+C_{\delta}\right)$; then, by (2), $D_{\delta} \subset a+B_{2 \delta}$ and $\left[w, \phi_{a}(w)\right] \subset \Omega \backslash \Lambda_{a}$ for any $w \in \partial D_{\delta}$. Since $\overline{M \backslash D_{\delta}} \subset \Omega \backslash \Lambda_{a}$ with $\partial\left(M \backslash D_{\delta}\right)=\partial M \cup \partial D_{\delta}$, by Theorem 3 we obtain $\int_{\partial M} F_{a}(w)=\int_{\partial D_{\delta}} F_{a}(w)$. Set $M_{1}=$ $\bigcup_{w \in \partial D_{\delta}}\left[w, \phi_{a}(w)\right]$; then since $M_{1} \subset \Omega \backslash \Lambda_{a}$ with $\partial M_{1}=\partial D_{1} \cup\left(a+\partial C_{\delta}\right)$, by Theorem 3 we obtain 


$$
\int_{\partial D_{\delta}} F_{a}(w)=\int_{a+\partial C_{\delta}} F_{a}(w)=\int_{\partial C_{\delta}} F_{a}(a+w) .
$$

Since $T_{a} M \in \mathscr{G}_{2}^{2 \lambda}\left(C^{2} \backslash \Lambda_{0}\right)$, by Theorem 1 (ii) there exists a smooth arc $W:[0,1] \rightarrow$ $\mathscr{G}_{2}^{2 \lambda}\left(\mathbf{C}^{2} \backslash \Lambda_{0}\right)$ such that $W(0)=T_{a} M$ and $W(1)=\lambda \mathbf{C e}_{0}$. Set $E_{\delta}(t)=B_{\delta} \cap W(t), S_{\delta}=E_{\delta}(1)$ and $M_{2}=\bigcup_{0 \leqq t \leqq 1} \partial E_{\delta}(t)$; then since $a+M_{2} \subset \Omega \backslash \Lambda_{a}$ with $\partial M_{2}=\partial C_{\delta} \cup \partial S_{\delta}$, by Theorem 3 we obtain

$$
\int_{\partial C_{\delta}} F_{a}(a+w)=\frac{\lambda}{2 \pi i} \int_{\partial S_{\delta}} \frac{f(a+w)}{w} d w .
$$

Since $S_{\delta} \subset \lambda \mathrm{Ce}_{0}$, from Cauchy's integral formula of complex analysis we find that the right side is equal to $f(a)$. On the same line of argument it is proved that Cauchy's integral formula holds in a neighborhood of $a$ in $M^{\circ}$. Furthermore, since $f$ is complex right regular in $\Omega$ and since $\int_{\partial M} F_{z}(w)$ is complex right regular in $D_{\Omega}(\partial M, a)$, Corollary 4 implies the assertion.

Q.E.D.

THEOREM 5 (Cauchy's Integral Formula). Let $p, q \in \mathbf{N}_{0}$ with $p \leqq q, \lambda= \pm 1, \Omega$ be a domain of $\mathbf{C}^{p+1} \subset \mathbf{C}_{p}, f: \Omega \rightarrow \mathbf{C}_{q}$ be complex right regular and $a \in \Omega$.

(i) For odd p let $M$ be a real $(p+1)$-dimensional piecewise smooth compact connected oriented submanifold of $\Omega$ with boundary such that $a \in M^{\circ}, M$ is smooth at a, $M \cap \Lambda_{a}=\{a\}$ and $T_{a} M \in \mathscr{G}_{p+1}^{\lambda}\left(\mathbf{C}^{p+1} \backslash \Lambda_{0}\right)$.

(ii) For even $p$ let $M$ be a real $(p+1)$-dimensional piecewise smooth compact connected oriented submanifold of $\Omega_{a}$ with boundary such that $a \in M^{\circ}, M$ is smooth at $a$, $M \cap \Lambda_{a}=\{a\}$ and $T_{a} M \in \mathscr{G}_{p+1}^{\lambda}\left(V_{0}^{p+1} \backslash \Lambda_{0}\right)$.

Then

$$
f(z)=\frac{\lambda}{\left|S^{p}\right|} \int_{\partial M} f(w) d \sigma_{w} \frac{1}{(w-z)\langle w-z\rangle^{p-1}} \quad \text { for any } z \in D_{\Omega}(\partial M, a),
$$

where $1 /(w-z)$ is the inverse element of a Clifford number $w-z \in \mathbf{C}^{p+1} \subset \mathbf{C}_{p}$.

Proof. By $F_{z}(w)$ we denote the integrand in the right side of the formula. We use the same notation as used in the proof of Theorem 4; then by argument analogous to the proof of Theorem 4 we obtain

$$
\int_{\partial M} F_{a}(w)=\int_{\delta D_{\delta}} F_{a}(w)=\int_{a+\partial C_{\delta}} F_{a}(w)=\int_{\partial C_{\delta}} F_{a}(a+w) .
$$

Since $T_{a} M \in \mathscr{G}_{p+1}^{\lambda}\left(\mathbf{C}^{p+1} \backslash \Lambda_{0}\right)$ for odd $p$, by Theorem 1 (iii) there exists a smooth arc $W:[0,1] \rightarrow \mathscr{G}_{p+1}^{\lambda}\left(\mathbf{C}^{p+1} \backslash \Lambda_{0}\right)$ such that $W(0)=T_{a} M$ and $W(1)=\lambda \mathbf{R}^{p+1}$. Since $T_{a} M \in$ $\mathscr{G}_{p+1}^{\lambda}\left(V_{0}^{p+1} \backslash \Lambda_{0}\right)$ for even $p$, by Theorem 1 (vi) there exists a smooth arc $W$ : $[0,1] \rightarrow \mathscr{G}_{p+1}^{\lambda}\left(V_{0}^{p+1} \backslash \Lambda_{0}\right)$ such that $W(0)=T_{a} M$ and $W(1)=\lambda \mathbf{R}^{p+1}$. Then by Theorem 3 we obtain 


$$
\int_{\partial C_{\delta}} F_{a}(a+w)=\frac{\lambda}{\left|S^{p}\right|} \int_{\partial S_{\delta}} f(a+w) d \sigma_{w} \frac{1}{w|w|^{p-1}} .
$$

Since $S_{\delta} \subset \lambda \mathbf{R}^{p+1}$, from Cauchy's integral formula of real Clifford analysis (see [2, §9]) we find that the right side is equal to $f(a)$. On the same line of argument it is proved that Cauchy's integral formula holds in a neighborhood of $a$ in $M^{\circ}$. Furthermore, since $f$ is complex right regular in $\Omega$ and since $\int_{\partial M} F_{z}(w)$ is complex right regular in $D_{\Omega}(\partial M, a)$, Corollary 4 implies the assertion.

Q.E.D.

REMARK 1. Theorems 4 and 5 are generalizations of Ryan's results in [5].

Remark 2. Let $q \in \mathbf{N}$ with $2 \leqq q, \Omega$ be a domain of $\mathbf{C}_{2}\left(\cong \mathbf{C}^{4}\right)$ and $f: \Omega \rightarrow \mathbf{C}_{q}$ be complex right regular, that is, $\sum_{j=0}^{3} \mathbf{e}_{j}\left(\partial f / \partial z_{j}\right)=0$, where $\mathbf{e}_{3}=\mathbf{e}_{1} \mathbf{e}_{2}$. Then Cauchy's integral formula also holds for this function $f$.

Proposition 14. Let $p \in \mathbf{N}$ be even, $q \in \mathbf{N}$ with $p \leqq q, \Omega$ be a domain of $\mathbf{C}^{p+1} \subset \mathbf{C}_{p}$, $f: \Omega \rightarrow \mathrm{C}_{q}$ be complex right regular with $f \neq 0, a \in \Omega$ and $M$ be a real $(p+1)$-dimensional piecewise smooth compact connected oriented submanifold of $\Omega_{a}$ with boundary such that $a \in M^{\circ}, M$ is smooth at $a, M \cap \Lambda_{a}=\{a\}$ and $T_{a} M \in \mathscr{G}_{p+1}^{\lambda}\left(V_{0}^{p+1} \backslash \Lambda_{0}\right)$. Then there is a closed submanifold $L$ of $\partial M$ such that $\langle w-z\rangle$ is single-valued in $L \times D_{\Omega}(L, a)$. Furthermore,

$$
f(z)=\frac{\lambda}{\left|S^{p}\right|} \int_{L} f(w) d \sigma_{w} \frac{1}{(w-z)\langle w-z\rangle^{p-1}} \quad \text { for any } z \in D_{\Omega}(L, a) .
$$

Proof. Since $M$ is a compact submanifold of $\mathbf{C}^{p+1}$ with boundary, $\partial M$ has at most finite number of components. By $F_{z}(w)$ we denote the integrand in the right side of the formula. Let $\Gamma$ be the set of all the loops $\gamma:[0,1] \rightarrow D_{\Omega}(\partial M, a)$ such that $\gamma(0)=\gamma(1)=a$. For $w, w^{\prime} \in \partial M$ we define an equivalence relation as

$$
w \sim w^{\prime} \Leftrightarrow \frac{\langle w-\gamma(1)\rangle}{\langle w-\gamma(0)\rangle}=\frac{\left\langle w^{\prime}-\gamma(1)\right\rangle}{\left\langle w^{\prime}-\gamma(0)\right\rangle} \text { for any loop } \gamma \in \Gamma .
$$

If $w$ and $w^{\prime}$ belong to the same component of $\partial M$, then $w \sim w^{\prime}$. Let $N_{1}, N_{2}, \cdots, N_{n}$ be the equivalence classes; then we obtain $\partial M=N_{1}+N_{2}+\cdots+N_{n}$. For any $\gamma \in \Gamma$ there is a submanifold $N^{\prime}$ of $\partial M$ such that $\langle w-\gamma(0)\rangle=\langle w-\gamma(1)\rangle$ for any $w \in N^{\prime}$ and such that $\langle w-\gamma(0)\rangle=-\langle w-\gamma(1)\rangle$ for any $w \in \partial M \backslash N^{\prime}$. From Theorem 5 it follows that

$$
\begin{gathered}
f(a)=\int_{\partial M} F_{a}(w)=\int_{N^{\prime}} F_{\gamma(0)}(w)+\int_{\partial M \backslash N^{\prime}} F_{\gamma(0)}(w) \\
=\int_{N^{\prime}} F_{\gamma(1)}(w)+\int_{\partial M \backslash N^{\prime}} F_{\gamma(1)}(w) .
\end{gathered}
$$

Therefore, we find that 


$$
f(a)=\int_{N^{\prime}} F_{a}(w), \quad \int_{\partial M \backslash N^{\prime}} F_{a}(w)=0 .
$$

By the definition of $\left\{N_{j}\right\}$ we obtain loops $\gamma_{1}, \gamma_{2}, \cdots, \gamma_{m} \in \Gamma$ and unions $N^{1}, N^{2}, \cdots, N^{m}$ of $N_{j}$ such that

$$
\begin{gathered}
\partial M \supset N^{1} \supset N^{2} \supset \cdots \supset N^{m}, N^{j} \neq N^{j+1}, \\
f(a)=\int_{\partial M} F_{a}(w)=\int_{N^{1}} F_{a}(w)=\cdots=\int_{N^{m}} F_{a}(w), \\
\int_{\partial M \backslash N^{1}} F_{a}(w)=\int_{N^{1} \backslash N^{2}} F_{a}(w)=\cdots=\int_{N^{m-1} \backslash N^{m}} F_{a}(w)=0 .
\end{gathered}
$$

The above equations also holds in a neighborhood of $a$ in $M^{\circ}$; furthermore, by Corollary 4 they hold in $D_{\Omega}(\partial M, a)$. If $N^{m}=\varnothing$, then $f=0$; however, this is contrary to our assumption $f \neq 0$. Thus, we obtain $N^{m}=\varnothing$; then $N^{m}=N_{j}$ for some $j, j=1, \cdots, m$. For simplicity, we set $j=1$. Then for any $w \in N_{1}$ and $\gamma \in \Gamma$ we find that $\langle w-\gamma(0)\rangle=\langle w-\gamma(1)\rangle$; therefore, $\langle w-z\rangle$ is single-valued in $N_{1} \times D_{\Omega}(\partial M, a)$. Moreover, we infer that

$$
\begin{gathered}
f(z)=\int_{N_{1}} F_{z}(w), \\
\int_{N_{j}} F_{z}(w)=0 \quad(j=2, \cdots, n) \quad \text { for any } z \in D_{\Omega}(\partial M, a) .
\end{gathered}
$$

Next, we consider loops in $D_{\Omega}\left(N_{1}, a\right)$ and repeat the same argument; further, repeat this. Then we finally obtain a closed submanifold $L$ of $\partial M$ which satisfies the conditions.

Q.E.D.

REMARK 1. If $p=0$ or if $\partial M$ is connected, then $\langle w-z\rangle$ is single-valued in $\partial M \times D_{\Omega}(\partial M, a)$.

Remark 2. Let $\Omega=\mathbf{C}^{p+1}$ and $M$ be included in an affine space $X$ in $\mathbf{C}^{p+1}$; then $L$ includes the boundary of the only unbounded domain of $X \backslash M$.

Remark 3. Let $p \in \mathbf{N}_{0}$ be even, $\Omega$ be a domain of $\mathbf{C}^{p+1}$ and $a \in \Omega$. If $f$ is complex regular in $\Omega \backslash \Lambda_{a}$, then $f$ is complex regular at $a$. If $f$ is not complex regular at $a$, then $f$ is multiple-valued; for example, Cauchy's kernel $1 / z\langle z\rangle^{p-1}$ is double-valued in $\mathbf{C}^{p+1} \backslash \Lambda_{0}$ (see [7, Corollary 3]).

\section{References}

[1] B. Avanissian, Cellule d'Harmonicité et Prolongement Analytique Complexe, Travaux en Cours, Hermann (1985). 
[2] F. Brackx, R. Delanghe and F. Sommen, Clifford Analysis, Research Notes in Math. 76, Pitman (1982).

[3] J. BUREక̌ and V. SouČEK, Generalized hypercomplex analysis and its integral formulas, Complex Variables Theory Appl. 5 (1985), 53-70.

[4] J. Ryan, Complexified Clifford analysis, Complex Variables Theory Appl. 1 (1982), 119-149.

[5] J. RYAN, Special functions and relations within complex Clifford analysis I, Complex Variables Theory Appl. 2 (1983), 177-198.

[6] J. RYAN, Singularities and Laurent expansions in complex Clifford analysis, Appl. Anal. 16 (1983), 33-49.

[ 7] K. Sano, Cauchy's integral formula in complex Clifford analysis, Simon Stevin 65 (1991), 293-317.

[8] V. SouČEK, Complex-quaternionic analysis applied to spin- $\frac{1}{2}$ massless fields, Complex Variables Theory Appl. 1 (1983), 327-346.

Present Address:

Department of Architecture, Hachinohe Institute of TeChNology, Myo ÔBIRAKI, HACHINOHE, AOMORI-KEN, 031 JaPAN. 